\title{
Clinical Research Nurses Perspective on Recruitment Challenges and Lessons Learnt from a Large Multi-site Observational Study
}

\section{Abstract \\ Background}

Recruitment of large numbers of study participants within a designated time frame for multisite clinical research studies is a significant challenge faced by researchers. If a study doesn't manage to recruit targeted number of participants, it could have a significant impact on the statistical significance of the research.

\section{Purpose}

This paper highlights the challenges of recruitment for a large multi-site UK based tuberculosis observational study 'PREDICT'.

\section{Methods}

It uses a case study analysis from the research nurses perspective, and descriptive information retrieved from non-recruitment log forms to understand reasons for potential recruits not participating.

\section{Results}

Some of the main challenges to recruitment included patients not attending their clinic appointments, time required to obtain site specific permissions, and courier timings for blood sample collection. This paper also outlines key reasons for potential recruits who did not participate. Some of the common barriers to participation for non-recruited participants were work and family commitments, additional blood tests and language barriers.

\section{Conclusions}


Successful strategies which were implemented to overcome some of the challenges during the study are presented. This paper, therefore, aims to present the challenges faced, lessons learnt, and successful strategies implemented to inform the planning of similar longitudinal studies of this scale in future.

Keywords: Research nurse, perspective, challenges to recruitment, multi-site observational study, Tuberculosis 


\section{Introduction}

Clinical research studies' success is based primarily on recruitment numbers due to the emphasis placed on generating statistical significance and generalisability. However, there is substantial evidence that many of these studies often achieve a low enrolment of participants within the targeted time frame and with the funding awarded (McDonald et al., 2006; Sully, Julious, \& Nicholl, 2013; Thoma, Farrokhyar, McKnight, \& Bhandari, 2010). If a study fails to recruit the required numbers of participants which were originally estimated to be enrolled (sample size) at the pace required, financial sponsors can lose interest and faith which can lead to funding withdrawal or no additional money to support the study further. Due to this, expected outputs can lose significance as other successful competing studies are completed and disseminated. Incomplete studies can cause an enormous waste of resources, knowledge, and time, and fail to add to the evidence base (Thoma et al., 2010; Walters et al., 2017). Slow recruitment and consequently delaying the results of studies is considered unethical as it potentially postpones any benefit that may arise from the prompt publication and dissemination of findings. It is therefore important to gain an understanding of the causes behind recruitment failure and how they can be avoided.

\section{Background}

This paper is based on the experiences gained from a large UK based, multi-site, latent tuberculosis (TB) cohort study 'Prognostic Value of Interferon Gamma Release Assays and Tuberculin Skin Test in Predicting the Development of Active Tuberculosis: The UK PREDICT TB Cohort Study' (Abubakar et al., 2018) which aimed to evaluate the predictive value of tests for latent tuberculosis. The study planned to recruit 10,000 participants from 25 National Health Service (NHS) hospitals and a network of General Practices across London during a 24 month period. The study participants were required to undertake tests for latent 
tuberculosis including a TB skin test (Mantoux screening test) and two interferon gamma release assays (blood tests), other relevant blood tests, and to complete a questionnaire. Participant follow up was for an average of 24 months using the national register of clinical reports, national microbiological database and a phone call at 12 and 24 months post recruitment. The eligibility criteria included anyone above the age of 16 years who had been in contact with someone with active TB (pulmonary or extra-pulmonary) during the previous six months; people who had entered the UK from a high prevalence TB country during the last five years; and people who had frequently travelled to high prevalence TB countries during the last five years [minimum stay of 12 months in the included country list] (World Health Organisation, 2018).

The study commenced participant recruitment in March 2011 from various TB clinics based at multiple London NHS hospitals. However, the recruitment during the targeted time period was slower than expected (490 participants were recruited during the first seven months). Figure 1 displays the rate of participant recruitment during the first eight months of the study recruitment period. The study finally accomplished the desired sample size of 10,000 participants in June 2015 (more than double the time that was originally estimated).

A review undertaken to explore factors which influence recruitment into Randomised Controlled Trials (RCT's) in the UK identified that failure to accomplish recruitment targets is a common concern amongst multi-site trials funded by large funding bodies (McDonald et al., 2006). Another UK based cancer research study explored challenges of recruitment in the context of a multi-centre cohort study (Fenlon et al., 2013). For this trial, the identification of participants was established by NHS colorectal cancer pre-surgical clinics who opted to participate in this study and the recruitment of participants was undertaken by NHS Trust based research nurses. They recruited over a thousand patients over seventeen months from thirty centres appointing local nurses as the Principle Investigators (PI). In contrast, the 
PREDICT study, approached multiple NHS TB clinics inviting them to participate, asking medical leads to take on the responsibility as local leads. The research nurses were recruited externally and some help was sought by local NHS Trust based research nurses.

The PREDICT study's targeted sample size was ten times higher than Fenlon et al's (2013) study with a considerably smaller recruitment time frame of twenty four months. No additional literature could be identified that addresses issues related to the recruitment of participants to large multi-site cohort observational studies offering practical solutions and strategies, even though many multi-site cohort observational studies are funded annually (NIHR, 2017/2018). This paper focuses on the challenges presented and lessons learnt during the PREDICT study to add to the research recruitment literature. It also presents strategies to consider in setting up future studies of this scale. This paper, therefore, aims to add to the knowledge base for future studies to draw on and improve recruitment numbers in a timely and efficient manner.

[Insert Figure 1.]

\section{Methods}

A case study analysis was used to identify recruitment issues from the research nurses perspective. Three research nurses (AI, SM, SMW) contributed to the case study drawing on their experience working on the PREDICT study. In addition, descriptive data is presented from the nurses' non-recruitment log forms. These logs were completed during the study to capture potential participants, who didn't consent to participate or who initially consented but later didn't fulfil participation requirements. The logs consisted of eleven non-participation statements (Figure 2) which the nurses completed after the potential recruits had advised on their reason for not participating. No further data was collected from the participants to 
ensure the logs were anonymous. The log categories were: not interested/no reason given; took information away (for consideration but did not participate); can't wait (declined upfront); Needle phobic; could not complete test (insufficient blood to fill the vials); showed interest but didn't wait (agreed but left before seen to); other; language barrier; likely to be lost to follow up (due to personal circumstances such as no fixed abode); put off by amount of blood; religious / moral reasons

\section{Results}

The results are split into three sections: Organisational challenges; Reasons provided from the non-recruited participants; and Strategies employed to improve the recruitment rate. These were the key areas identified by the research nurses within the case study that had an impact on recruitment.

\section{Organisational challenges}

The following headings highlight the key organisational challenges that were a barrier to recruiting participants from a research nurse perspective. Each theme is discussed in detail.

\section{Patients attendance}

TB contact clinics (nurse-led clinics in the UK that organise the screening of individuals who have been in contact with someone diagnosed with TB) frequently suffered from patients who did not attend (DNA) appointments. This considerably affected the number of potential participants research nurses could approach and was a significant reason for slower recruitment (MacLellan et al., 2016). In addition, some patients who were booked into the contact clinics were not necessarily eligible for the study. For example, patients referred from 
other diagnostic clinics for TB screening. This was one of the major barriers for recruiting participants faced by the research nurses working on the study.

\section{Staff recruitment and site specific permissions}

The research nurses employed on the study were appointed on short, fixed term contracts. Due to this, the research study experienced frequent turnover of staff. Furthermore, a research passport (to enable the research nurses to work in various NHS hospitals) was required for all research nurses linked to the study, which took considerable time to obtain due to multiple criminal records checks having to be conducted and a lack of joined-up working. These were significant issues which affected the number of nurses that were available and able to recruit study participants.

\section{Courier and laboratory timings and providers}

Courier travel timings were calculated based on the timings of the blood samples collected from the study site to drop off to the laboratory for analysis. Each laboratory had a cut off time in which samples had to be received by. Due to heavy congestion on the roads during peak rush hours, collection timings often had to be brought forward to ensure specimens arrived on time, this meant that the time available for recruitment was cut short. In addition, sometimes there were delays in the courier sample collection which had implications on whether samples could be accepted by the contracted laboratory or had to be re-routed to an alternative laboratory (that had later working hours), adding additional costs. The study was restricted to using services from approved contracted couriers only; hence, it was not possible to obtain these services from another provider.

\section{Travelling between recruitment sites}

Research nurses were required to travel between multiple sites to recruit participants from various TB clinics across London. This wasn't always practical depending on the number of participants recruited during the morning clinic (packaging of blood samples and 
documentation required extra time); clinics running late, couriers running late, geographical distances between the clinics, and timing issues related to public transport and traffic. For this reason, the research nurses were often not able to provide full cover to either morning or afternoon clinics and had to prioritise sites. However, multi-site coverage by PREDICT study research nurses was identified over time as the most productive way to recruit higher numbers in comparison to the numbers NHS Trust based research nurses could recruit.

\section{TB clinic timings}

In some hospitals, TB nurse-led contact clinics were shorter than anticipated. This limited the time for the research nurses to recruit eligible participants. Most of the TB clinics were arranged during a half-day slot - morning or afternoon. Some TB clinics would finish in less than two hours due to DNAs or patients returning for results only. Furthermore, in most cases, there were two TB nurses working together to see patients simultaneously. Conversely, only one research nurse was usually assigned to cover a clinic and required an average of 20 minutes to recruit a participant. Due to this, research nurses often missed opportunities to approach and recruit eligible participants.

Other issues experienced at the TB clinics included a lack of support for the research from local hospital staff members, space and storage issues on sites, and various other TB studies also recruiting for eligible participants from the same clinics with a similar eligibility criteria.

\section{Reasons provided from the non-recruited participants}

A non-recruitment log form was maintained over a period of a year, commencing at the start of the study but not continuing for the duration of the study due to staff turnover. In total 533 responses were captured. The log form asked non-recruits of their reasons for not participating. However, not everyone gave a reason for non-participation and therefore the respondents' rate was low. Hence, the cumulative results of these log forms did not seem to 
be fully representative of what the research nurses experienced in practice (Figure 2). The following headings merge the outcome of the responses with the research nurses perspectives in regards to non-participation.

\section{Time required to participate}

In most cases, research nurses were lone working which would require additional waiting time for patients who were keen on taking part. Some of these potential participants weren't willing to wait for the blood tests due to work or other commitments, and therefore the speed and efficiency of the research nurses, and the number of research nurses on site was a factor in recruitment numbers.

\section{Volume of blood required and needle phobia}

The research required patients to provide approximately $29 \mathrm{mls}$ of blood for study related tests. Some patients required additional blood tests for their TB clinical management which could be ranging from 3-15mls. This often increased their reluctance to participate due to the perceived volume of blood required. Some patients were needle phobic and therefore declined participation.

\section{Language barriers}

A smaller number of participants weren't recruited due to language barriers. In anticipation of this, a language phone line was made available. However, due to the length of time it could take to communicate through the language phone line, participants often lost interest and declined to complete the questionnaire.

\section{Incentives}

The study awarded $£ 10$ per participant to compensate for their time and travel. Initially the incentives were controlled centrally, which meant that a voucher would be posted to the 
participant after recruitment which could take some weeks. Although attractive to participants, the reward wasn't instant and therefore not as appealing as anticipated.

\section{Cultural factors and personal beliefs}

In certain cases, the head of a family would be the decision-maker in regards to the participation of family members. In those situations, a number of potential participants would be lost if the decision of the head was against participation. Some participants expressed concerns regarding their values and beliefs around the handling and storing of blood samples.

This was an infrequent reason for declining to participate. However, during certain time periods we experienced a steep reduction in the number of recruits due to observance of religious practices.

[Insert Figure 2.]

\section{Strategies employed to improve the recruitment rate}

The study team managed to introduce and adopt strategies which proved to be successful in the long run for the effective and efficient recruitment of participants. The following describes the strategies in more detail.

\section{Smarter travelling between sites}

PREDICT study research nurses travelling between sites to cover clinics was successful in the recruitment of participants in comparison to adding recruitment to the pre-existing studies portfolio of NHS Trust based research nurses. However, it still posed issues due to clinic and courier timings. To increase the likelihood of research nurses arriving on time to clinics, the active recruitment clinics were mapped out and aligned to public transport routes, generating a more efficient placement of the research nurses. Moreover, the research nurses offered 
flexibility in terms of their working hours and distances commuted to accommodate study coverage to the clinics.

\section{Adjusting courier timings}

Due to the limitations of using approved courier services, the study was not able to employ an alternative service. To solve this challenge, the study management team adjusted the collection timings (learning through trial and error) in order to avoid problems of late delivery to the laboratory. The employment of this strategy ensured the samples were collected and dropped off on time.

\section{Opening new sites}

After carrying out an initial analysis of sites which were effectively and efficiently recruiting, it was decided to establish other recruitment sites within the same communities. Therefore, the recruitment of the study was further extended to local places of worships, places of work, educational institutions, and to primary care General Practices. Recruitment at primary care General Practices didn't achieve a good number of participants, however, recruitment at places of worships and work places became the most successful strategy employed to improve numbers.

Further research nurses were recruited for being bi/multilingual which proved successful when the study was extended into communities where the majority of recruits were nonEnglish speakers.

\section{Relationship building}

Another significant strategy was communication and relationship building with local TB nurses alongside other members within their team. At some sites, PREDICT research nurses faced challenges in terms of a lack of support by local teams. However, overtime working alongside TB clinic nurses and staff, acceptance and co-working was established by sharing workloads. For example, the research nurses began offering to perform clinically prescribed 
blood tests alongside the research blood tests they were conducting. This approach not only established a relationship but also saved the TB clinic nurses time resulting in the clinic nurses offering to help with the study treatment follow up. Alongside this, TB clinic nurses also helped in sorting out space issues for storage and consultation.

In addition to the above, research nurses were assigned to work together on busy sites to capture more potential recruits.

\section{Incentives}

The Participant compensation of $£ 10$ in vouchers which was initially controlled centrally and sent post-recruitment was improved by allowing the research nurses to offer the vouchers instantly. Voucher distribution was monitored by requiring study recruits to sign (alongside a voucher identification number) for the vouchers they received.

\section{Discussion}

For longitudinal large scale multi-site studies it is often challenging to predict the barriers to recruitment in advance and hence most of the challenges discussed above were inevitable. However, similarly to any project in a healthcare setting, proper planning for longitudinal multi-site observational studies at the research design phase can save time, resources and effort. According to Thoma and colleagues "Investigators significantly overestimate the pool of available patients who meet the inclusion criteria" (Thoma et al., 2010). For PREDICT, It was estimated that from 12 different clinics, 7 patients would be recruited per clinic each week to achieve the targeted sample size of 10,000 participants during a 24 month period. This was calculated on the basis of patients' attendance at contact clinics in two hospital sites over a short period and projected to be accurate for the whole of London over a longer time period. This was clearly not the case. In addition, TB contact clinics are not restricted to the attendance of study eligible patients only, which meant that the estimated numbers were not a true representation of the eligible patients who attended the clinics. Hence, the original target 
to recruit 10,000 participants in 24 months was found to be extremely optimistic and therefore unachievable. The amount of DNA's that each clinic received was also not factored into the calculations.

Multi-disciplinary team and stakeholder involvement is crucial during the planning phase of a study. This should include but is not limited to medical doctors, scientists, nurses, healthcare assistants, managers and patient representatives. Substantial networking and communication with local healthcare teams and collaboration with relevant research teams could aid in making the ground work more robust, particularly in relation to multi-site studies. In addition, performing a well-planned pilot study before commencing the main study and incorporating any learning into the study could help in forecasting the correct estimates for recruitment.

Before rolling out a study to multiple sites, research investigators should aim to educate all the stakeholders and healthcare professionals involved in the study regarding the goals and potential outcomes of the research in order to gain their full support. They could also arrange periodic feedback meetings to further strengthen the relationships. Principle investigators should liaise with other researchers planning to undertake similar studies to ensure that there is no competition for the same study population that could reduce recruitment numbers for all parties.

For research nurses, there should be further incentives offered to retain staff. For example, continuing educational development opportunities along with well-structured career pathways, longer-term contracts, and the opportunity to be more involved in research at all stages of the process (not just data collection). A structured career pathway for research nurses in general would improve research nurse recruitment, retention, skills and knowledge. From a practical perspective, Research and Development (R\&D) departments should aim to shorten the time period required for letters of access and honorary research contracts, aiming to streamline the process by working collaboratively with other healthcare organisations. For 
the collection of samples, flexibility in the laboratory timings and a firm commitment from the courier services would improve sample numbers immensely. Incentives should be provided directly to participants during recruitment, not only to increase recruits but to save administrative time and costs. Consideration should also be given to religious practices and how they may effect recruitment due to fasting and prayer timings, and widely held beliefs about research. Where possible, investigators should identify the languages spoken of the study population and aim to recruit research nurses who possess these skills.

\section{Limitations}

This paper would have benefited from a more systematic approach of recording the various causes of low recruitment for a greater length of time in order to make the findings more robust and applicable for future studies in similar contexts. We also didn't record the number of potential participants who declined to participate in this sub study. In addition, further qualitative research exploring the causes of low recruitment from the research nurses point of view and reluctance to participate from the participants' side would have been useful in exploring the issues further and identifying potential solutions. Future studies would benefit from including this data as part of the overall research strategy.

\section{Conclusions}

Recruitment of participants is a challenge for any clinical research study. It requires input from a range of stakeholders prior to conducting a pilot study to increase the successfulness of the study objectives. This paper has drawn on the practical experience of research nurses and frequent reasons from potential recruits who chose not to participate in a UK-based study. Based on this case study, we have provided recommendations to improve recruitment of observational multi-site cohort studies during the study design phase, the implementation 
phase, and during the recruitment time itself. We hope these insights will be of use to those undertaking future observational multi-site cohort studies and subsequently will reduce waste in terms of resources, time and effort and successively speed up the research outcomes and its potential to be impactful. For future researchers, this information can help them to address these challenges in the planning process of the study to avoid under recruitment.

\section{Key points:}

1) It is vital to involve stakeholders and multi-disciplinary team representatives at the planning stage of recruitment. This paper aims to help researchers in creating realistic goals of recruiting participants for large clinical trials.

2) Education and engagement with key professionals from various sites during the planning stage could prove successful in recruiting potential participants.

3) For large, multi-site, longitudinal studies, the total number of potential recruits should be calculated on the basis of eligible patients who attend the clinic on each site for a minimum of four to eight weeks to observe attendance trends and patterns, taking into account DNAs (Did Not Attend; patients/participants who did not attend their appointment) and realistic numbers of participants who are willing and able to participate in the study.

4) Incentives in the form of opportunities for continuing professional development and engagement forums (e.g. staff away days) could help to motivate research nurses who work in isolation. Regular physical or remote meetings to discuss recruitment strategies could help to recruit higher numbers through shared experiences.

\section{Ethics permission}

The study procedures and protocol were approved by the Brent NHS Research Ethics Committee (10/H0717/14). 


\section{References}

Abubakar, I., Drobniewski, F., Southern, J., Sitch, A. J., Jackson, C., Lipman, M., . . . Lalvani, A. (2018). Prognostic value of interferon-gamma release assays and tuberculin skin test in predicting the development of active tuberculosis (UK PREDICT TB): a prospective cohort study. Lancet Infect Dis. doi:10.1016/s1473$3099(18) 30355-4$

Fenlon, D., Seymour, K. C., Okamoto, I., Winter, J., Richardson, A., Addington-Hall, J., .. . Foster, C. (2013). Lessons learnt recruiting to a multi-site UK cohort study to explore recovery of health and well-being after colorectal cancer (CREW study). BMC Med Res Methodol, 13, 153. doi:10.1186/1471-2288-13-153

MacLellan, J., Wallace, K., Vacchelli, E., Roe, J., Davidson, R., Abubakar, I., \& Southern, J. (2016). A multi-perspective service evaluation exploring tuberculosis contact screening attendance among adults at a North London hospital. Journal of Public Health, 38(3), e362-e367.

McDonald, A. M., Knight, R. C., Campbell, M. K., Entwistle, V. A., Grant, A. M., Cook, J. A., ... Snowdon, C. (2006). What influences recruitment to randomised controlled trials? A review of trials funded by two UK funding agencies. Trials, 7, 9. doi:10.1186/1745-6215-7-9

NIHR, N. I. f. H. R. (2017/2018). Key statistics Retrieved from https://www.nihr.ac.uk/about-us/how-we-are-managed/managing-centres/crn/keystatistics.htm

Sully, B. G. O., Julious, S. A., \& Nicholl, J. (2013). A reinvestigation of recruitment to randomised, controlled, multicenter trials: a review of trials funded by two UK funding agencies. Trials, 14(1), 166. doi:10.1186/1745-6215-14-166 
Thoma, A., Farrokhyar, F., McKnight, L., \& Bhandari, M. (2010). Practical tips for surgical research: how to optimize patient recruitment. Can J Surg, 53(3), 205-210.

Walters, S. J., Bonacho Dos Anjos Henriques-Cadby, I., Bortolami, O., Flight, L., Hind, D., Jacques, R. M., . . Julious, S. A. (2017). Recruitment and retention of participants in randomised controlled trials: a review of trials funded and published by the United Kingdom Health Technology Assessment Programme. BMJ Open, 7(3), e015276. doi:10.1136/bmjopen-2016-015276

World Health Organisation. (2018). World Health Organization (WHO) estimates of tuberculosis incidence by country, 2016. Retrieved from https://www.gov.uk/government/uploads/system/uploads/attachment_data/file/677927 /WHO_estimates_of_tuberculosis_incidence_by_country_2016.pdf

\section{Figures caption}

Figure1. Total number of participants recruited over eight months from various NHS hospital Trusts

Figure 2. Data collected from participants who declined recruitment for the PREDICT study 